\title{
Técnica de interligação extracapsular fêmoro-fabelo-tibial na ruptura do ligamento cruzado cranial em cães: achados clínicos e radiográficos
}

\author{
Extracapsular femoro-fabelo-tibial inter-connection technique used in dogs with cranial cruciate ligament \\ rupture-clinical and radiographic findings
}

\author{
Durval Baraúna JúniorI Cláudio Roehsig ${ }^{\mathrm{II}}$ Leandro Branco Rocha ${ }^{\mathrm{II}}$ Ricardo Chioratto ${ }^{\mathrm{III}}$ \\ Eduardo Alberto Tudury ${ }^{\mathrm{IV}}$
}

\section{RESUMO}

O objetivo deste trabalho foi avaliar os resultados da técnica de interligação extracapsular fêmoro-fabelo-tibial, como forma de tratamento da ruptura do ligamento cruzado cranial (RLCC) espontânea em cães, utilizando para isto avaliações clínicas e radiográficas. Neste estudo, foram operados 13 cães, divididos em dois grupos: ruptura unilateral $(R U)$ e ruptura bilateral (RB). O peso variou entre 2,3 e $53 \mathrm{~kg}$ (mediana $6,7 \mathrm{~kg}$ ) e o intervalo entre a suspeita da lesão observada pelos proprietários e os procedimentos cirúrgicos variaram entre três e 365 dias (média de 123 dias). Em nenhum dos 13 joelhos operados foram observadas complicações transoperatórias. Após 30 dias de pós-operatório, os dois grupos apresentaram diferença estatisticamente significante $(P=0,05)$ nos parâmetros funcionais avaliados, com exceção da dor $e$ disposição para levantar o membro contralateral no grupo de cães com ruptura bilateral. Ao exame radiográfico nos dias zero e 180, a evolução das alterações degenerativas foi observada em $84,6 \%$ dos casos. A técnica de interligação extracapsular fêmoro-fabelo-tibial é eficiente na estabilização imediata da articulação com RLCC, oferecendo recuperação funcional ao reduzir o deslocamento cranial e a rotação interna da tíbia, porém não impede a progressão da osteoartrite.

Palavras-chave: cirurgia, ortopedia, joelho, cão.

\section{ABSTRACT}

This study was aimed at evaluating the results of extracapsular femoro-fabelo-tibial inter-connection technique, for the treatment of spontaneous cranial cruciate ligament rupture (CCLR) in dogs, using for this purpose clinical and radiographic evaluation. In this study 13 dogs allocated in two groups were operated: unilateral rupture $(R U)$ and bilateral rupture (RB). The weight ranged between 2.3 and $53 \mathrm{~kg}$ (median
$6.7 \mathrm{~kg}$ ) and the interval between the suspect of lesion observed by the owners and the surgical procedures ranged between three and 365 days (mean 123 days). None of the operated knee were observed intra-operative complication. After 30 days of postoperative the two groups showed statistically significant difference $(P=0.05)$ in the evaluated parameters, except of the pain and willingness to hold up the contralateral limb on the group of the dogs with bilateral rupture. At the radiographic exam on the 0 and 180 days, the evolution of degenerative changes was seen in $84.6 \%$ of cases. The extracapsular femurfabelo-tibial interconnection technique is efficient in the immediate stabilization of joint with CCLR, offering functional recovery for reduce the cranial displacement and internal rotation of the tibia, however it doesn't stop the osteoarthritis progression.

Key words: surgery, orthopedics, stifle joint, dog.

\section{INTRODUÇÃO}

Em 1926, Carlin relatava pela primeira vez a ruptura do ligamento cruzado cranial (RLCC) na medicina veterinária e, somente no ano de 1952, Paatsama desenvolveu a primeira técnica de reconstituição desse ligamento em cães. Desde então, inúmeros procedimentos cirúrgicos foram e vêm sendo pesquisados (ARNOCZY, 1985). Tratamento conservador ou cirúrgico são as opções, porém a controvérsia em relação à escolha da melhor conduta persiste (PIERMATTEI \& FLO, 1999). Em cães com menos de $25 \mathrm{~kg}$, a terapia conservativa pode ser

\footnotetext{
IColegiado de Medicina Veterinária, Universidade Federal do Vale do São Francisco (UNIVASF), 56300-900, Petrolina, PE, Brasil. E-mail: durvalbarauna@hotmail.com. Autor para correspondência.

IIPrograma de Pós-graduação em Ciência Veterinária, Universidade Federal Rural de Pernambuco (UFRPE), Recife, PE, Brasil.

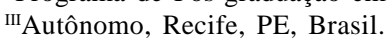

${ }^{\text {IV }}$ Departamento de Medicina Veterinária, UFRPE, Recife, PE, Brasil.
} 
suficiente; embora o procedimento cirúrgico reduza a atrofia muscular e as alterações degenerativas, que invariavelmente ocorrem com o método conservador (LEVINE et al., 2004). A cirurgia é recomendada para todos os cães, pois acelera a taxa de recuperação e potencializa a função (SCHWARZ, 2003).

CONZEMIUS et al. (2005) observaram que, em cães de raças grandes, a sutura extracapsular de estabilização lateral e a osteotomia niveladora do platô tibial (TPLO) apresentaram resultados similares e superiores à estabilização intra-articular “over-the-top”. Para CHAUVET et al. (1996), quando a facilidade do procedimento e a satisfação do cliente são os objetivos do cirurgião, parece que a sutura fabelo-tibial é a melhor opção para cães grandes com RLCC; quando a meta é o pleno retorno da função e a prevenção da doença articular degenerativa, nenhum procedimento cirúrgico é satisfatório.

O sistema de grampo tubular de aço inoxidável, indicado para prender os fios nas técnicas de sutura fabelo-tibial, tem o potencial de eliminar a irritação provocada pelo nó, provendo ao cirurgião uma mais consistente tensão inicial, menor alongamento e maior resistência à ruptura, quando comparado ao nó convencional (ANDERSON III et al., 1998).

SILVA (2004) e TUDURY et al. (2005) descreveram duas novas técnicas para correção da RLCC similares quanto à interligação óssea entre o fêmur e a crista tibial, que são de extrema simplicidade, baixo custo e com bons resultados, mas com mínimas diferenças entre elas. Independentemente do procedimento cirúrgico, a progressão da osteoartrite ocorre e os achados radiográficos pioram (CHIERICHETTI, 2001), porém de forma mais lenta, quando comparada com a articulação de cães não tratados cirurgicamente (BIASI et al., 2002).

O objetivo deste trabalho foi avaliar os resultados da técnica de interligação extracapsular fêmoro-fabelo-tibial, como forma de tratamento da RLCC espontânea em cães, utilizando para isto avaliações clínicas e radiográficas.

\section{MATERIAL E MÉTODOS}

Foram utilizados 13 cães portadores de RLCC, selecionados durante o atendimento de rotina do Hospital Veterinário da UFRPE e divididos em dois grupos, um composto por oito cães com ruptura unilateral operados (RU) e outro por cinco cães com ruptura bilateral operados unilateralmente (RB) (Tabela 1). Exames radiográficos de todos os joelhos afetados nas projeções mediolateral sob estresse e craniocaudal antes da cirurgia foram úteis tanto na confirmação da suspeita da lesão quanto na avaliação das alterações degenerativas articulares de acordo com a classificação (ausente, discreta, moderada e severa) estabelecida por MOORE \& READ (1995).

No dia da cirurgia e conforme aprovado pela Comissão de Ética do Departamento de Medicina Veterinária da UFRPE, os cães foram submetidos a anestesia, profilaxia antimicrobiana (cefalotina sódica ${ }^{\mathrm{a}}$ intravenosa), anti-sepsia e colocação rotineira dos campos cirúrgicos. A técnica cirúrgica consistiu de abordagem medial ao joelho, para remoção dos restos

Tabela 1 - Identificação do cão; joelho afetado, lado operado e o tipo de ruptura; claudicação, suporte do peso, dor e disposição para levantar o membro contralateral (DPLMCL) aos dias 0, 30, 90 e 180 dias de pós-operatório; diâmetro em milímetros do fio de náilon (Ø) utilizado para estabilização e tempo de evolução (TE). Recife - 2006.

\begin{tabular}{|c|c|c|c|c|c|c|}
\hline Identificação do cão & $\begin{array}{l}\text { Tipo de } \\
\text { ruptura }\end{array}$ & Claudicação & $\begin{array}{l}\text { Suporte do } \\
\text { peso }\end{array}$ & Dor & DPLMCL & $(\varnothing) / \mathrm{TE}$ \\
\hline 1 - Shar Pei, 2a e 6m, 22 kg, F & (RU) & $(4 / 2 / 1 / 1)$ & $(4 / 3 / 1 / 1)$ & $(3 / 2 / 2 / 1)$ & $(2 / 2 / 2 / 1)$ & $1,2 / 2 \mathrm{~m}$ \\
\hline 2 - Poodle, 9a, 10 kg, M & $(\mathrm{RU})$ & $(3 / 1 / 1 / 1)$ & $(3 / 1 / 1 / 1)$ & $(3 / 1 / 1 / 1)$ & $(3 / 3 / 1 / 1)$ & $0,7 / 4 \mathrm{~m}$ \\
\hline 3 - Poodle, 8a, 6,7 kg, F & $(\mathrm{RU})$ & $(4 / 3 / 1 / 1)$ & $(4 / 5 / 1 / 1)$ & $(3 / 2 / 1 / 1)$ & $(3 / 3 / 1 / 1)$ & $0,7 / 2 \mathrm{~m}$ \\
\hline 4 - SRD, 7a,18 kg, F & $(\mathrm{RU})$ & $(4 / 2 / 1 / 1)$ & $(5 / 2 / 1 / 1)$ & $(2 / 2 / 1 / 1)$ & $(4 / 3 / 1 / 1)$ & $1,0 / 1 \mathrm{~m}$ \\
\hline 5 - Poodle, 6a, 6,7 kg, F & (RU) & $(4 / 2 / 1 / 1)$ & $(3 / 2 / 1 / 1)$ & $(2 / 1 / 1 / 1)$ & $(3 / 2 / 1 / 1)$ & $0,7 / 3 \mathrm{~m}$ \\
\hline 6 - Poodle, 1a e $10 \mathrm{~m}, 5,6 \mathrm{~kg}, \mathrm{M}$ & (RU) & $(5 / 1 / 1 / 1)$ & $(4 / 1 / 1 / 1)$ & $(2 / 1 / 1 / 1)$ & $(5 / 1 / 1 / 1)$ & $0,7 / 3 d$ \\
\hline 7 - Yorkshire, 8a, 5,7kg, F & (RU) & $(2 / 2 / 1 / 1)$ & $(2 / 2 / 1 / 1)$ & $(2 / 1 / 1 / 1)$ & $(3 / 2 / 1 / 1)$ & $0,7 / 1 \mathrm{a}$ \\
\hline 8 - Poodle, 8a, 2,7 kg, F & $(\mathrm{RU})$ & $(2 / 1 / 1 / 1)$ & $(2 / 1 / 1 / 1)$ & $(2 / 1 / 1 / 1)$ & $(2 / 1 / 1 / 1)$ & $0,5 / 3 \mathrm{~m}$ \\
\hline 9 - Yorkshire, 4a, 2,3 kg, F & $(\mathrm{RB})$ & $(4 / 3 / 2 / 1)$ & $(5 / 4 / 1 / 1)$ & $(2 / 1 / 1 / 1)$ & $(5 / 4 / 2 / 1)$ & $0,5 / 4 d$ \\
\hline 10 - Poodle, 7a, 4,0 kg, F & $(\mathrm{RB})$ & $(2 / 1 / 1 / 1)$ & $(2 / 1 / 1 / 1)$ & $(1 / 1 / 1 / 1)$ & $(4 / 1 / 1 / 1)$ & $0,5 / 1 \mathrm{~m}$ \\
\hline 11 - Pit Bull, 2a e 6m, 27 kg, M & $(\mathrm{RB})$ & $(3 / 2 / 1 / 1)$ & $(2 / 1 / 1 / 1)$ & $(1 / 1 / 1 / 1)$ & $(3 / 1 / 1 / 1)$ & $1,4 / 3 \mathrm{~m}$ \\
\hline 12 - Fila, 4a, 53 kg, M & $(\mathrm{RB})$ & $(3 / 2 / 1 / 1)$ & $(2 / 1 / 1 / 1)$ & $(2 / 1 / 1 / 1)$ & $(3 / 2 / 1 / 1)$ & $1,8 / 1 \mathrm{a}$ \\
\hline 13 - Fila, 5a, 43 kg, M & (RB) & $(4 / 2 / 1 / 1)$ & $(3 / 2 / 1 / 1)$ & $(2 / 2 / 1 / 1)$ & $(2 / 2 / 1 / 1)$ & $1,8 / 1 \mathrm{~m}$ \\
\hline
\end{tabular}

a - ano; m - mês; d- dia; M - macho; F - fêmea; RU - ruptura unilateral; RB - ruptura bilateral ; SRD - sem raça definida. 
de ligamento cruzado cranial (LCC), remoção de osteófitos peri-trocleares, intercondilares e patelares, avaliação da presença de alterações degenerativas e a inspeção dos meniscos. Em seguida, houve a sutura da membrana sinovial com categute cromado ${ }^{\mathrm{b}} 3-0$, em padrão contínuo simples e da cápsula articular com padrão interrompido simples, utilizando fio de náilon monofilamentarc ${ }^{c}$.

A técnica de interligação extracapsular fêmoro-fabelo-tibial (Figura 1) constou de duas perfurações ósseas utilizando-se pinos de Steinmann de diferentes diâmetros, acoplados a uma furadeira de baixa rotação; uma na crista tibial (distal e caudal à inserção do ligamento patelar) e a outra perfurando os côndilos femorais (cranial e distal às fabelas), em sentido transversal do lado medial para o lateral. Dois fios de náilon de pesca (autoclavados a $120^{\circ} \mathrm{C}$ durante 30 minutos) foram passados e amarrados através do orifício da crista tibial: um para sutura fabelo-tibial (f1) e o outro para a interligação fêmoro-tibial (f2). Uma das pontas do f2 foi passada em direção ao orifício condilar lateral e a outra em direção ao orifício condilar medial (sob as fáscias musculares profundas e sobre a cápsula articular). Com ajuda de uma agulha introduzida do orifício lateral e emergindo no orifício medial, a ponta de f2 do lado medial foi introduzida na agulha e tracionada através do orifício criado nos côndilos femorais, até aparecer no lado lateral. As duas pontas do f1 foram passadas por baixo da fáscia lata na direção da fabela lateral. Estando as quatro pontas presentes no lado lateral, foi realizada primeiramente a sutura fabelo-tibial (Figuras 1 Ae B), conforme recomendações de SCHAEFER \& FLO (1998). Em seguida, foi realizada a interligação óssea femoro-tibial com um assistente mantendo o joelho em um ângulo de $135^{\circ}$, reduzindo todo o deslocamento tibial cranial e desfazendo a rotação medial da crista tibial, o cirurgião realizava a amarra após tração manual às pontas do $\mathrm{f} 2$ (Figuras $1 \mathrm{C}$ e D), com seis seminós. Nos casos de fios de grosso calibre e de difícil maleabilidade, foi utilizado um grampo tubular ${ }^{\mathrm{d}}$ composto de aço inoxidável 316L, com diâmetro luminar adequado, que ao ser esmagado com alicate de corte, fixava as extremidades do fio sob tração. A aproximação do tecido subcutâneo foi executada com sutura contínua simples, com fio de categute cromado e a síntese da pele com pontos simples isolados com náilon monofilamentar. Em seguida, colocou-se bandagem protetora com atadura de crepom.

A escolha do diâmetro do fio de poliamidae (náilon de pesca) a ser empregado seguiu as

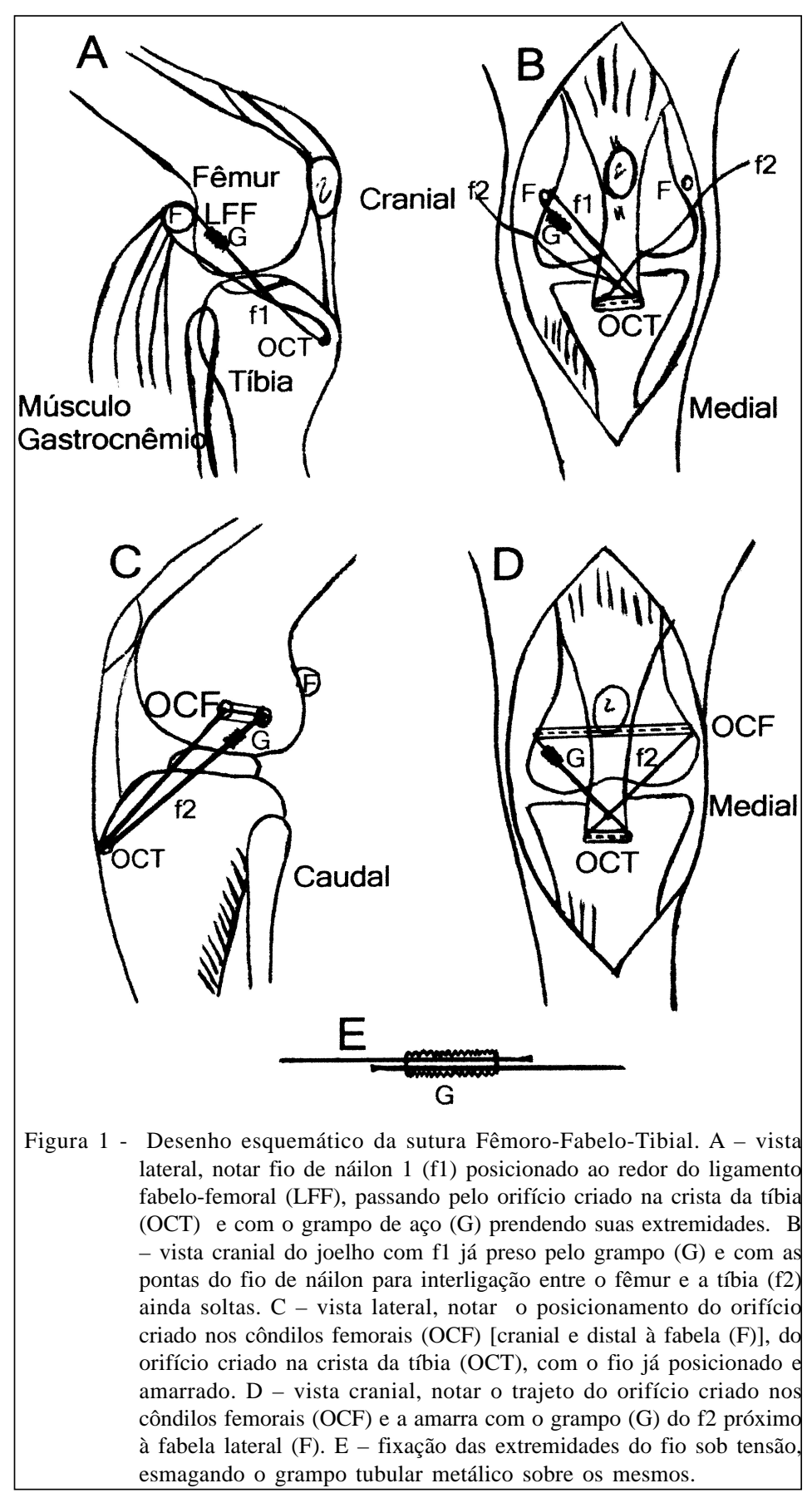

Ciência Rural, v.37, n.3, mai-jun, 2007. 
recomendações de resistência de acordo com os valores dos testes realizados por BARAÚNA JR. et al. (2005), sendo a resistência igual ou superior ao peso do animal.

Após a recuperação anestésica, os cães foram entregues aos proprietários com prescrição de meloxican (0,1mg kg-1, SID, PO) durante cinco dias, cefalexina (25mg kg-1, TID, PO) durante sete dias, sulfato de condroitina A (SID, PO) durante 60 dias e doxiciclina (3 a $5 \mathrm{mg} \mathrm{kg}^{-1}$, BID, PO) durante seis semanas. No pós-operatório imediato, foi recomendado também que eles seguissem o seguinte regime fisioterápico: gelo por cinco minutos de seis em seis horas, por 48 horas, e movimentos passivos de flexão e extensão do joelho (30 repetições de oito em oito horas por quatro semanas). Na quarta semana, recomendou-se iniciar com caminhadas curtas na coleira, aumentando gradualmente o tempo.

Os animais foram acompanhados no précirúrgico (dia zero), e no pós-operatório aos 30, 90 e 180 dias, através de exames específicos: rotação interna da tíbia (RIT), movimento de gaveta cranial (MGC) em extensão e flexão, movimento de gaveta caudal, teste de compressão tibial, efusão articular, e quantificação do perímetro da coxa em local pré-fixado e constante. A escala funcional proposta por GEELS et al. (2000) foi utilizada para avaliar os graus de claudicação, o suporte do peso do corpo, a disposição para levantar o membro contralateral e a presença de dor; acompanhando a evolução antes e após a cirurgia. A avaliação radiográfica foi repetida, após seis meses da estabilização cirúrgica, verificando-se, ao final desse período, progressão ou não das alterações articulares degenerativas.

Para a análise estatística dos parâmetros funcionais, foi utilizado o teste não-paramétrico de Wilcoxon, para diferenças entre pares ordenados. Para tal, utilizou-se o programa estatístico SPSS 12 , sendo considerados significantes os valores de $\mathrm{P} \leq 0,05$. Para a avaliação do perímetro da coxa, as médias foram comparadas através do teste de análise de variância (ANOVA- critério único), sendo considerados significativos os valores de $\mathrm{P} \leq 0,05$ (SAMPAIO, 1998).

\section{RESULTADOS E DISCUSSÃO}

Para SCHAEFER \& FLO (1998), mesmo os fios grossos sendo de difícil manuseio, quando o náilon de pesca monofilamentar é usado, a resistência do material selecionado deve aproximar-se ao peso do animal. No presente estudo, porém, não foram observadas dificuldades no manuseio dos fios de diâmetros superiores a 1,00mm ou na fixação do náilon, fosse ela por meio de nós ou com grampos tubulares. Não ocorreu ruptura do fio, nem afrouxamento da estabilização por falha técnica, do nó ou do grampo, em nenhum dos animais avaliados, apesar de ter sido observado por BUQUERA et al. (2002) afrouxamento da sutura anti-rotacional e soltura do fio ao redor da fabela, devido à fixação inadequada dos mesmos no ligamento fabelo-femoral. O fio de náilon de pesca é um material de baixo custo, monofilamentar, fácil de encontrar e esterilizar, disponível em diferentes diâmetros, e apresenta baixa taxa de infecção (SICARD et al., 1999), por isso, foi o material de escolha neste experimento. O fio de poliéster recomendado por SILVA(2004), para estabilização da RLCC, não foi opção neste experimento por estar associado a aumento da incidência de infecção (SMITH, 2000). Além disso, a resistência fornecida pelo fabricante é de $13 \mathrm{~kg}$, o que inviabilizaria a sua utilização em animais de grande porte.

Dos 13 cães avaliados (Tabela 1), a mediana de peso foi de $6,7 \mathrm{~kg}$ (média 15,9 kg). O intervalo entre a suspeita da lesão por parte dos proprietários e os procedimentos cirúrgicos variaram entre três e 365 dias, (média 123 dias). SINGLETON (1969) citou que, independentemente do porte do animal, entre 42 e 56 dias após a RLCC, já existem sinais macroscópicos moderados de artrite e, a partir de 56 dias, sinais avançados desta, fato visto durante a artrotomia exploratória na maioria dos cães deste experimento.

Dos 13 joelhos operados, em dois (1 e 13) foi detectado um grau discreto (até $2 \mathrm{~mm}$ ) de instabilidade no MGC e no teste de compressão tibial, nas avaliações pós-operatórias até 180 dias (Tabela 2); entretanto, estes cães não apresentaram comprometimento funcional no período de avaliação. Estes achados corroboram BUDSBERG et al. (1988), CHAUVET et al. (1996) e CHIERICHETTI et al. (2001), que também observaram a presença de discreto MGC em suas investigações, sem que isso fosse um fator determinante para o insucesso clínico. O discreto MGC pode ser explicado também pelas observações de HUBER et al. (1999), SICARD et al. (1999) e SMITH (2000), citando que o náilon tem a característica de poder afrouxar ou falhar como resultado de distensão pela sua elasticidade, deformação plástica, aperto e deformação dentro do nó.

Em outros dois cães (3 e 4), um grau discreto de MGC permaneceu até o trigésimo dia, desaparecendo aos 90 dias. Este fato pode ser explicado pelas características das técnicas extra-articulares, que, em longo prazo, geram o espessamento periarticular necessário à estabilidade articular em resposta ao 
Técnica de interligação extracapsular fêmoro-fabelo-tibial na ruptura do ligamento cruzado cranial em cães...

Tabela 2 - Resultados das avaliações ortopédicas nos joelhos operados e não operados com RLCC. Rotação interna da tíbia (RIT), Movimento de gaveta cranial em extensão (MGCE) e flexão (MGCF), Teste de compressão tibial (TCT) e Perímetro da coxa aos dias 0, 30, 90 e 180 dias de pós-operatório. Avaliação radiográfica das alterações articulares degenerativas (AAD) nos dias 0 e 180 após o procedimento cirúrgico. Recife - 2006.

\begin{tabular}{|c|c|c|c|c|c|c|}
\hline Joelho & RIT & MGCE & MGCF & TCT & Perímetro da coxa & $\mathrm{AAD}$ \\
\hline 1 & $(+/-/-/-)$ & $(+/ \mathrm{D} / \mathrm{D} / \mathrm{D})$ & $(+/ D / D / D)$ & $(+/ D / D / D)$ & $(31 / 30 / 32 / 34.5)$ & $\mathrm{D}-\mathrm{M}$ \\
\hline 1 & & & & & $(37 / 37 / 35.5 / 36)$ & \\
\hline 2 & $(+/ D /-/-)$ & $(+/ D /-/-)$ & $(+/ D /-/-)$ & $(+/ D /-/-)$ & $(25 / 24 / 27 / 27)$ & $A-D$ \\
\hline 2 & & & & & $(24.5 / 26 / 26.5 / 26.5)$ & \\
\hline 3 & $(+/ \mathrm{D} /-/-)$ & $(+/-/-/-)$ & $(+/-/-/-)$ & $(+/-/-/-)$ & $(18 / 18.5 / 19.5 / 20)$ & $\mathrm{D}-\mathrm{M}$ \\
\hline 3 & $(+/+/+/+)$ & $(-/-/-/ D)$ & $(-/-/-/ D)$ & $(-/-/-/ D)$ & $(21.5 / 24 / 22.5 / 22.5)$ & \\
\hline 4 & $(+/+/+/+)$ & $(-/-/-/-)$ & $(-/-/-/-)$ & $(-/-/-/-)$ & $(28.5 / 30 / 32.5 / 33.5)$ & \\
\hline 4 & $(+/ \mathrm{D} /-/-)$ & $(+/ D /-/-)$ & $(+/-/-/-)$ & $(+/-/-/-)$ & $(27.5 / 29 / 31 / 32)$ & $\mathrm{M}-\mathrm{M}$ \\
\hline 5 & & & & & $(22.5 / 23.5 / 22.5 / 22.5)$ & \\
\hline 5 & $(+/-/-/-)$ & $(+/-/-/-)$ & $(+/-/-/-)$ & $(+/-/-/-)$ & $(22.5 / 20 / 20 / 21.5)$ & $A-D$ \\
\hline 6 & $(+/-/-/-)$ & $(+/-/-/-)$ & $(+/-/-/-)$ & $(+/-/-/-)$ & $(21.5 / 21.5 / 21.5 / 21.5)$ & $A-D$ \\
\hline 6 & & & & & $(21.5 / 22.5 / 22 / 22)$ & \\
\hline 7 & $(\mathrm{D} / \mathrm{D} / \mathrm{D} / \mathrm{D})$ & $(-/-/-/-)$ & $(-/-/-/-)$ & $(-/-/-/-)$ & $(18 / 18 / 20 / 22)$ & \\
\hline 7 & $(+/-/-/-)$ & $(+/-/-/-)$ & $(+/-/-/-)$ & $(+/-/-/-)$ & $(18 / 18 / 18 / 18)$ & $\mathrm{D}-\mathrm{D}$ \\
\hline 8 & $(+/-/-/-)$ & $(+/-/-/-)$ & $(+/-/-/-)$ & $(+/-/-/-)$ & $(14 / 14.5 / 15.5 / 16)$ & $D-M$ \\
\hline 8 & $(+/+/+/+)$ & $(-/-/-/-)$ & $(-/-/-/-)$ & $(-/-/-/-)$ & $(15 / 16 / 16 / 16)$ & \\
\hline 9 & $(+/-/-/-)$ & $(+/-/-/-)$ & $(+/-/-/-)$ & $(+/-/-/-)$ & $(14.5 / 15.5 / 14 / 15)$ & $\mathrm{D}-\mathrm{M}$ \\
\hline 9 & $(+/+/+/+)$ & $(-/-/+/+)$ & $(-/-/+/+)$ & $(-/-/+/+)$ & $(14.5 / 16 / 15 / 15)$ & \\
\hline 10 & $(+/-/-/-)$ & $(+/-/-/-)$ & $(+/-/-/-)$ & $(+/-/-/-)$ & $(18.5 / 19 / 19 / 19)$ & $A-D$ \\
\hline 10 & $(+/+/+/+)$ & $(+/+/+/+)$ & $(+/+/+/+)$ & $(+/+/+/+)$ & $(18.5 / 17.5 / 18 / 18)$ & \\
\hline 11 & $(+/+/+/+)$ & $(+/+/+/+)$ & $(+/+/+/+)$ & $(+/+/+/+)$ & $(41 / 42 / 42 / 42)$ & \\
\hline 11 & $(+/-/-/-)$ & $(+/-/-/-)$ & $(+/-/-/-)$ & $(+/-/-/-)$ & $(39 / 39.5 / 43 / 43)$ & $M-S$ \\
\hline 12 & $(+/+/+/+)$ & $(+/+/+/+)$ & $(+/+/+/+)$ & $(+/+/+/+)$ & $(49 / 46.5 / 46 / 47)$ & \\
\hline 12 & $(+/-/-/-)$ & $(+/-/-/-)$ & $(+/-/-/-)$ & $(+/-/-/-)$ & $(48 / 48 / 48 / 48)$ & $\mathrm{M}-\mathrm{S}$ \\
\hline 13 & $(-/-/-/-)$ & $(\mathrm{D} / \mathrm{D} / \mathrm{D} / \mathrm{D})$ & $(-/-/-/-)$ & $(+/-/-/-)$ & $(43.5 / 44.5 / 44.5 / 44)$ & \\
\hline 13 & $(+/-/-/-)$ & $(+/ \mathrm{D} / \mathrm{D} / \mathrm{D})$ & $(+/ D / D / D)$ & $(+/ D / D / D)$ & $(44.7 / 42 / 43 / 43.5)$ & $\mathrm{D}-\mathrm{M}$ \\
\hline
\end{tabular}

D - discreto; A - ausente; M - moderada; S- severa; + positivo; - negativo.

procedimento cirúrgico (MOORE \& READ, 1996). Outra provável explicação para esse desaparecimento do MGC é que a fisioterapia pós-cirúrgica possa ter tornado a articulação mais estável, conforme citaram LEVINE et al. (2004).

As médias da avaliação funcional (claudicação, dor, suporte do peso e disposição para levantar o membro contralateral) foram decrescentes nos dois grupos operados até 180 dias de pósoperatório. Os animais dos dois grupos apresentaram recuperação estatisticamente significante $(\mathrm{P}=0,05)$ aos 30 dias pós-operatório na claudicação e suporte do peso, com evolução estatisticamente significante até 90 dias. A avaliação da dor no grupo RU exibiu, aos 30 dias de pós-operatório, diferença estatisticamente significante; já no grupo RB, não houve diferença estatística entre os tempos. Relacionado à disposição para levantar o membro contralateral, o grupo RU apresentou resultado superior ao grupo RB, em que o primeiro apresentou diferença estatisticamente significante a partir dos 30 dias de pós-operatório com evolução até 90 dias, enquanto o segundo apresentou diferença estatisticamente significante, somente após 90 dias de pós-operatório (Tabela 3).

Acredita-se que os bons resultados obtidos, como recuperação funcional de em média três meses e uma taxa de eficiência de $100 \%$, decorreram do procedimento cirúrgico, principalmente nos animais de pequeno porte (menos de 15kg) que não apresentaram evolução satisfatória com terapia conservadora, apesar de o tempo médio entre a suspeita da lesão e a intervenção cirúrgica ter sido de 16 semanas, diferentemente do esperado por PIERMATTEI \& FLO (1999), que relataram a possibilidade de recuperação, independente do procedimento cirúrgico. VASSEUR (2003) citou que, em cães de pequeno porte, é prudente esperar pelo menos 6 a 8 semanas antes de recomendar a cirurgia, fato observado neste experimento em virtude do tempo esperado pelos proprietários até levarem seus cães ao atendimento.

Ciência Rural, v.37, n.3, mai-jun, 2007. 
Tabela 3 - Média e desvio padrão dos graus de claudicação, suporte de peso pelo membro afetado, presença de dor, disposição para levantar o membro contralateral e perímetro da coxa dos joelhos operados e não operados aos 0, 30, 90 e 180 de pós-operatório, dos joelhos com RLCC. Recife - 2006.

\begin{tabular}{|c|c|c|c|c|}
\hline Eventos & Dia 0 & Dia 30 & Dia 90 & Dia 180 \\
\hline Claudicação(RU) & $3,6( \pm 0,9)$ aA & $1,9( \pm 0,6) \mathrm{bB}$ & $1,0( \pm 0,0) \mathrm{cC}$ & $1,0( \pm 0,0) \mathrm{cD}$ \\
\hline Claudicação(RB) & $3,2( \pm 0,8)$ aA & $2,0( \pm 0,7) \mathrm{bB}$ & $1,2( \pm 0,4) \mathrm{cC}$ & $1,0( \pm 0,0) \mathrm{bcD}$ \\
\hline Suporte do peso (RU) & $3,4( \pm 1,1)$ aA & $2,1( \pm 1,4) \mathrm{bB}$ & $1,0( \pm 0,0) \mathrm{cC}$ & $1,0( \pm 0,0) \mathrm{cD}$ \\
\hline Suporte do peso (RB) & $2,8( \pm 1,3)$ aA 1,0 & $1,8( \pm 1,3) \mathrm{bB}$ & $( \pm 0,0)$ bcC & $1,0( \pm 0,0) \mathrm{bcD}$ \\
\hline Dor (RU) & $2,4( \pm 0,5)$ aA & $1,4( \pm 0,5) \mathrm{bB}$ & $1,1( \pm 0,4)$ bcC & $1,0( \pm 0,0) \mathrm{bcD}$ \\
\hline Dor (RB) & $1,6( \pm 0,5)$ aA & $1,2( \pm 0,4) \mathrm{aB}$ & $1,0( \pm 0,0) \mathrm{aC}$ & $1,0( \pm 0,0) \mathrm{aD}$ \\
\hline $\begin{array}{l}\text { Disposição para levantar membro } \\
\text { contra-lateral (RU) }\end{array}$ & $3,1( \pm 1,0)$ aA & $2,1( \pm 0,8) b B$ & $1,1( \pm 0,4) \mathrm{cC}$ & $1,0( \pm 0,0) \mathrm{cD}$ \\
\hline $\begin{array}{l}\text { Disposição para levantar membro } \\
\text { contra-lateral (RB) }\end{array}$ & $3,4( \pm 1,1)$ aA & $2,0( \pm 1,2) \mathrm{abB}$ & $1,2( \pm 0,4)$ bcC & $1,0( \pm 0,0)$ bcD \\
\hline Joelho operado (RU) & $22,2( \pm 5,6) \mathrm{aA}$ & $21,9( \pm 5,4) \mathrm{aB}$ & $23,1( \pm 6,2) \mathrm{aC}$ & $23,8( \pm 6,7) \mathrm{aD}$ \\
\hline Joelho não operado (RU) & $23,6( \pm 6,8)$ aA & $24,6( \pm 6,6) \mathrm{aB}$ & $24,7( \pm 6,5) \mathrm{aC}$ & $25,1( \pm 6,6) \mathrm{aD}$ \\
\hline Joelho operado (RB) & $32,94( \pm 15,4)$ aA & $32,8( \pm 14,6) \mathrm{aB}$ & $33,4( \pm 15,7)$ aC & $33,7( \pm 15,4) \mathrm{aD}$ \\
\hline Joelho não operado(RB) & $33,3( \pm 15,7)$ aA & $33,3( \pm 15,2)$ aB & $33,1( \pm 15,3) \mathrm{aC}$ & $33,2( \pm 15,4) \mathrm{aD}$ \\
\hline
\end{tabular}

Média ( \pm desvio padrão), $\mathrm{n}=8$ (ruptura unilateral), $\mathrm{n}=5$ (ruptura bilateral).

Médias seguidas das mesmas letras minúsculas, na mesma linha, e das mesmas letras maiúsculas na mesma coluna não diferem significantemente entre si $(\mathrm{P}>0,05)$.

RU -ruptura unilateral, RB - ruptura bilateral.

Quanto ao perímetro da coxa, todas as comparações entre os dois grupos testados e entre os tempos foram feitas através das médias e estatisticamente não foram detectadas diferenças significantes $(\mathrm{P}>0,05)$ (Tabela 3). A dor e o desuso do membro poderiam causar hipotrofia muscular (PIERMATTEI \& FLO, 1999). BIASI et al. (2002) observaram a manutenção do perímetro da coxa, naqueles animais que tiveram uma melhor função do membro após a correção cirúrgica.

Em relação à técnica cirúrgica, não foram observadas complicações transoperatórias. A escolha pela adição de uma sutura fabelo-tibial baseou-se no pré-experimento, no qual a RIT não foi eficientemente evitada pela técnica de fixação côndilo-tibial, sugerida por SILVA(2004). Segundo ANDERSON et al. (1998), as técnicas cirúrgicas devem evitar o deslocamento cranial da tíbia em relação ao fêmur e prevenir excessiva RIT.

Compressão anormal nas superfícies articulares decorrentes da sutura fabelo-tibial foi apontada como uma desvantagem biomecânica dos procedimentos extracapsulares (SMITH, 2000). No entanto, SELMI et al. (2003) demonstraram a eficiência da sutura fabelo-tibial utilizando fios com propriedades elásticas, na restituição da estabilidade do joelho após transecção do LCC, principalmente por manter o posicionamento do vetor velocidade tangente à superfície articular, não alterando assim a biomecânica normal dessa articulação, embora tenha ocorrido alteração do centro instantâneo de movimento (CIM) da posição extra-articular para uma posição intraarticular. Biomecanicamente, a técnica fêmoro-fabelotibial parece apresentar comportamento similar à sutura fabelo-tibial tradicional, em relação à análise do CIM e manutenção do vetor velocidade tangente à superfície articular. No entanto, nenhuma informação concreta sobre o procedimento pode ser afirmada, pois ainda não foram realizados estudos completos.

As avaliações biomecânicas dos procedimentos de reparo do LCC devem buscar tanto a estabilização do joelho quanto o grau de deslocamento cranial, como também a promoção de rigidez articular (ROMANO et al., 2006). Com base na análise destes parâmetros, a técnica descrita mostrouse eficaz ao inibir ou reduzir o deslocamento cranial da tíbia em relação ao fêmur.

De acordo com a classificação proposta por MOORE \& READ (1995), os exames radiográficos para acompanhamento da alteração articular degenerativa dos 13 joelhos avaliados mostraram que: no dia zero, quatro (31\%) foram classificadas em ausente, seis (46\%) em discreta, três (23\%) em moderada; e, aos 180 dias, cinco (38,5\%) em discreta, seis (46\%) em moderada e dois (15,5\%) em severa (Tabela 2). Esses dados permitem afirmar que houve evolução radiográfica das alterações degenerativas em $84,6 \%$ dos joelhos operados, mesmo instituindo um protocolo terapêutico (sulfato de 
condroitina e doxiciclina), no intuito de minimizar a progressão da doença articular degenerativa, conforme recomendação de VASSEUR (2003). Os resultados obtidos estão de acordo com BIASI et al. (2002) e VASSEUR (2003) que citaram não existir procedimento cirúrgico que evite piora dos achados radiográficos, devido à progressão da osteoartrite.

\section{CONCLUSÃO}

A técnica de interligação extracapsular fêmoro-fabelo-tibial é eficiente na estabilização imediata da articulação de cães com RLCC, oferecendo recuperação funcional ao reduzir o deslocamento cranial e a rotação interna da tíbia, porém não impede a progressão da osteoartrite.

\section{AGRADECIMENTOS}

À Coordenação de Aperfeiçoamento de Pessoal de Nível Superior (CAPES), pela bolsa de mestrado.

\section{FONTES DE AQUISIÇÃO}

a Cefalotina Sódica ${ }^{\circledR}$ - Eli Lilly do Brasil Ltda. Recife-PE. b Categute cromado $^{\circledR}$ - Cirumédica Brasil - Cotia - SP. c Mononylon ${ }^{\circledR}$ - Ethicon - Johnson \& Johnson Produtos Profissionais - São José dos Campos - SP.

d Grampos tubulares - Fermed Instrumental Médico Cirúrgico Ltda - BH - MG

e Grilon ${ }^{\circledR}$ - Mazzaferro Fishing. Indústria Brasileira.

\section{COMISSÃO DE ÉTICA}

Este trabalho foi aprovado pela Comissão de ética em experimentação animal do Departamento de Medicina Veterinária da UFRPE - Campus Dois Irmãos, sob número de processo 11231 -2004, estando de acordo com as normas do Colégio Brasileiro de Ética em Experimentação Animal (COBEA)

\section{REFERÊNCIAS}

ANDERSON III, C.C. et al. Biomechanical evaluation of crimp clamp system for loop fixation of monofilament nylon leader material used for stabilization of the canine stifle joint. Veterinary Surgery, Philadelphia, v.27, n.6, p.533-539, 1998.

ARNOCZY, S.P. Cruciate ligament rupture and associate injuries: In: NEWTON, C.D.; NUNAMAKER, D.M. Textbook of small animall orthopaedics. Philadelphia: Lippincott, 1985. p.403-414.

BARAÚNA JR. D. et al. Resistência dos diferentes tipos de fios indicados para estabilização de joelhos de cães com ruptura do ligamento cruzado cranial. In: JORNADA DE ENSINO, PESQUISA E EXTENSÃO DA UFRPE, 5., 2005, Recife, PE. Anais... Recife: UFRPE, 2005. 1CD.

BIASI, F. et al. Avaliação clínica e radiográfica da reconstrução do ligamento cruzado cranial em cães com uso de aloenxerto de ligamento patelar congelado. Ars Veterinária, Jaboticabal, v.18, n.2, p.100-106, 2002.

BUDSBERG, S.C. et al. Force plate analyses before and after stabiliation of canine stifles for cruciate injury. American Journal of Veterinary Research, Chicago, v.49, n.9, p.1522 $-1524,1988$.

BUQUERA, L.E.C. et al. Radiografia e macroscopia após estabilização extra-articular utilizando fáscia lata, fio de poliéster trançado ou fio de poliamida para correção da ruptura do ligamento cruzado cranial em cães. Ciência Rural, Santa Maria, v.32, n.1, p.73-78, 2002.

CHAUVET, A.E. et al. Evaluation of fibular head transposition, lateral suture, and conservative treatment of cranial cruciate ligament rupture in large dogs: a retrospective study. Journal of the American Animal Hospital Association, Lakewood, v.32, p.247-255, 1996.

CHIERICHETTI, A.L. et al. Ruptura de ligamento cruzado cranial. Estudo comparativo da técnica extra-articular com enxerto autógeno de fáscia lata com e sem artrotomia exploratória. Clínica Veterinária, São Paulo, n.33, p.3442, 2001.

CONZEMIUS, M.G. et al. Effect of surgical technique on limb function after surgery for rupture of the cranial cruciate ligament in dogs. Journal of the American Veterinary Medical Association, Schaumburg, v.226, n.2, p.232-236, 2005

GEELS, J.J. et al. Evaluation of intracapsular technique for the treatment of cranial cruciate ligament rupture. Veterinary and Comparative Orthopedics and Traumatology, Stuttgart, v.13, p.197-203, 2000.

HUBER, D.J. et.al. The effect of knotting method on the structural properties of large diameter nonabsorbable monofilament sutures. Veterinary Surgery, Philadelphia, v.28, p.260-267, 1999.

LEVINE, D. et al. Common orthopedic conditions and their physical reabilitation. In: MILLIS, D.L. et al. Canine reabilitation e physical therapy. St. Louis: Saunders, 2004. p.355-387.

MOORE, K.W.; READ, R.A. Cranial cruciate ligament rupture in the dog - a retrospective study comparing surgical techniques. Australian Veterinary Journal, Brunswich, v.72, p.281285, 1995.

MOORE K.W., READ, R.A. Rupture of cranial cruciate ligament in dogs - Part II. Diagnosis and management. Compendium on Continuing Education for the Practicing Veterinarian, Lawrenceville, v.18, n.4, p.381-391, 1996.

PIERMATTEI, D.L.; FLO G.L. A articulação fêmuro-tíbiopatelar (joelho). In:______. Manual de ortopedia e tratamento das fraturas dos pequenos animais. 3.ed. São Paulo: Manole, 1999. p.480-535.

ROMANO, L. et al. Análise biomecânica do joelho íntegro e com ruptura do ligamento cruzado cranial quanto ao grau de deslocamento cranial e rigidez articular em cães. Acta Cirúrgica Brasileira, São Paulo, v.21, p.46-50, 2006. 
SAMPAIO, I.B.M. Estatística aplicada à experimentação animal. Belo Horizonte: Fundação de Ensino e Pesquisa em Medicina Veterinária e Zootecnia, 1998. p.221.

SCHAEFER, S.L.; FLO, G.L. Cranial cruciate ligament repair by modified retinacular imbrication technique. In: BOJRAB, M.J. et al. Current techniques in small animal surgery. 4.ed. Baltimore: William \& Williams, 1998. p.1206-1209.

SCHWARZ, P.D. Doença do ligamento cruzado cranial. In: TILLEY, L.P.; SMITH, F.W.K. Consulta veterinária em 5 minutos. 2.ed. São Paulo: Manole, 2003. p.596-597.

SELMI, A.L. et al. Centro instantâneo de movimento, na avaliação ex-vivo da reconstrução extra-articular fabelo-tibial, após transecção do ligamento cruzado cranial em cães. Ciência Rural, Santa Maria, v.33, n.5, p.875-880, 2003.

SICARD, G.K. et al. Comparison of fishing line for repair of the cruciate deficient stifle. Veterinary and Comparative Orthopedics and Traumatology, Stuttgart, v.12, p.138-141, 1999.
SILVA, R.S. Estabilização da articulação femoro-tibiopatelar pós-ruptura do ligamento cruzado cranial pela técnica de fixação côndilo-tibial extra-articular em cães. 2004. 65f. Tese (Doutorado em Ciências Veterinárias, Sanidade Animal) - Universidade Federal Rural do Rio de Janeiro.

SINGLETON, W.B. Observations based upon the surgical rapair of 106 cases of anterior cruciate ligament rupture. Journal Small Animall Practice, London, v.10, p.269-278, 1969.

SMITH, B. Viewpoints in surgery cruciate ligament rupture extracapsular stabilisation. Australian Veterinary Journal, Brunswich, v.78, n.6, p.382-383, 2000.

TUDURY, E.A. et al. Interligação extracapsular femoro-fabelotibial: nova técnica de tratamento da ruptura do ligamento cruzado, em cães. Revista Anclivepa, São Paulo, n.3, p.9293, 2005.

VASSEUR, P.B. Stifle joint. In: SLATTER, D. Textbook of small animal surgery. 3.ed. Philadelphia: Saunders, 2003. Cap.147, p.2090-2133. 